\title{
Is working memory affective in dementia of alzheimer's type?
}

\author{
Nicola Mammarella ${ }^{1 *}$, Beth Fairfield ${ }^{1}$, Alberto Di Domenico' ${ }^{1}$ Erika Borella ${ }^{2}$ and Barbara Carretti ${ }^{2}$ \\ *Correspondence: n.mammarella@unich.it \\ 'Department of Psychological Sciences, University of Chieti, Italy. \\ ${ }^{2}$ Department of General Psychology, University of Padua, Italy.
}

\begin{abstract}
Background: The aim of the present study was to examine whether patients with Dementia of Alzheimer's Type (DAT) show a working memory emotional enhancement effect.

Methods: We used an affective version of the working memory operation span test that included negative and positive words as well as neutral ones (as in the classical version).

Results: Results showed that while healthy older adults performed better with emotional compared to neutral words, emotional effects were nullified in DAT patients.

Conclusions: Altogether, results indicate that DAT patients do not benefit from emotional cues when maintenance and manipulation of information in working memory are required.
\end{abstract}

Keywords: Dementia, emotions, working memory, alzheimer's type

\section{Introduction}

Working Memory (WM) can be described as a memory system that temporarily maintains and manipulates different types of information during a task at hand [1] and many experimental tasks have been developed to study its functions. Recently, attention has shifted to the role that emotions may have in modulating WM functions and different laboratory studies are focused on emotional effects in WM performance of older adults $[2,3]$. These studies have shown that, despite the welldocumented age-related decline in general resources and in WM $[4,5]$, age-related differences in WM may be attenuated when tasks include valenced words. It is, however, important to note that, in general, the tasks used to assess age-related differences in WM capacity for emotional stimuli differ with respect to the classical requests of a WM task. In fact, in a typical WM span tasks [6,7], participants are required to process (read and judge) a series of sentences, words or operations, maintain and remember final words/digits in their correct serial order. These tasks have been shown to entail concurrent processing and short-term storage demands coupled with an attentional control component to limit interference between processing and storage. On the contrary, most studies on emotion effects adopted more passive forms of WM tasks in which participants are basically required to remember affective information, words or pictures, without processing other information.

Therefore, whether there are emotion modulation effects in a classical WM span task and whether affective information can reduce age-related differences between young and older adults in a more active type of WM task are still open questions. Recently, a study by Mammarella and colleagues [2] showed that age-related differences were nullified when participants engaged in a modified version of the operation WM span test in which positive and negative valence words as well as neutral ones (as in the classical working memory task) are presented.

Moreover, the question of whether Dementia of Alzheimer's Type (DAT) patients differ from healthy older adults in terms of their ability to maintain and manipulate emotional information in an affective version of a classical WM task still needs to be clarified. In fact, a series of previous studies with DAT patients has yielded mixed results. For example, Kensinger and colleagues [8] in a sentence generation task and with verbal descriptions [9] found no emotional enhancement effect, while Fleming et al., [10] in an immediate recall task for emotional words and Satler and Tomaz [11] who tested DAT patients' ability to recognize emotional information in a visuo-spatial WM task found emotional benefits. Consequently, generalizing WM emotional enhancement effects in DAT must be taken with caution and it may be that results depend on the type of WM task used.

The goal of the present study was thus to assess whether WM difficulties in patients with Dementia of the Alzheimer's Type are reduced when actively manipulating affective information. To this end, we adopted a classical WM span task in which participants judged math operations during the processing phase and then had to actively maintain (maintenance phase) a set of unrelated positive or negative or neutral -as in the original task-, words in memory, and ultimately remember only final target words (affective and neutral ones). In line with literature [12], we expected to find a general decline in DAT patients' performance in the WM task compared to healthy older adults. Moreover, if DAT patients benefit from affective information in a task in which they must process information 
Mammarella et al. Neuroscience Discovery 2013,

http://www.hoajonline.com/journals/pdf/2052-6946-1-4.pdf

doi: 10.7243/2052-6946-1-4

Table 1. Means (M) and standard deviations (SD) of demographic and cognitive variables by age group.

\begin{tabular}{lllll}
\hline & \multicolumn{2}{l}{ Older (10F/10M) } & \multicolumn{2}{c}{ DAT (9F/11M) } \\
\hline & M & SD & M & SD \\
\hline Background & & & & \\
Age & 70.70 & 5.09 & 76.80 & 3.24 \\
Years of education & 7.65 & 3.07 & 6.15 & 1.23 \\
MMSE & 27.9 & 1.70 & 19.4 & 2.70 \\
Forward digit & 4.45 & 2.06 & 3.65 & 2.30 \\
Backward digit & 4.30 & 1.17 & 3.70 & 0.57 \\
PANAS positive & 30.95 & 3.99 & 23.55 & 4.29 \\
PANAS negative & 15.2 & 5.75 & 16.35 & 3.44 \\
\hline
\end{tabular}

Note. $\mathrm{F}=$ female participants, $\mathrm{M}=$ male participants.

while actively maintaining affective information, we expect these differences to be less pronounced when affective words are considered.

\section{Methods \\ Participants}

Twenty older adults (age range: $65-82$ years) and twenty DAT patients (age range: $70-82$ years) voluntarily took part in the experiment. In particular, healthy community-dwelling older adults were recruited from the Pescara-Chieti area. All healthy older adults reported being in good health, with no history of stroke, heart disease, primary degenerative neurological disorder or psychiatric disease. They had normal, or corrected to normal, vision and hearing (for inclusion criteria see Crook and colleagues [13]. The DAT patients, instead, met the criteria of AD described in the Diagnostic and Statistical Manual of Mental Disorders (Ed. $4^{\text {th }}$ ) by the American Psychiatric Association in 1994. Participants were recruited from local medical centers in the Abruzzo region and were examined by a neurologist and a geriatrician. A clinical diagnosis of $A D$ was determined for each patient. The severity of $A D$ ranged from mild to moderate (scores 1 or 2) according to the Clinical Dementia Rating Scale. All DAT patients had normal or corrected-to-normal vision and hearing. All participants were excluded if characterized by clinically significant depression or alcohol or drug use as assessed with a structured clinical interview, and if they had a cerebrovascular disease, or traumatic brain damage. Written informed consent was obtained from all participants and/or their caregivers (where appropriate). The study was approved by the Department of Psychological Sciences ethical committee (University of Chieti). All DAT patients were treated with reversible acetylcholinesterase inhibitors.

In addition to the experimental task, all participants took the Mini Mental State Exam [14] and performed the forward and backward version of the digit span (maximum score 8) [15]. The two groups differed significantly in MMSE scores ( 27.9 vs. $19.4, p<.001)$ but did not differ in terms of years of education and general cognitive abilities (forward and backward digit span) $F<1$. Moreover, no age-differences were found for the PANAS negative, while older adults were slightly more positive than DAT patients [16], (see Table 1 for descriptive statistics). None of the participants was paid for their participation.

\section{Material and procedure}

Operation working memory span (Ospan) test for emotional words (adapted from Turner and Engle [7]). The Ospan requires participants to solve a series of math operations (processing phase) while trying to remember a set of unrelated words (maintenance phase). Participants see one math operation/ word string at a time, centered on a computer monitor. For each trial, they read the math problem aloud, decide whether the given solution is true or false and then read the word aloud. Immediately after the participant reads the word, the next operation-word string is presented. The operation-word strings were presented in sets of three to six items. Following each complete set, participants were cued to recall target words in the correct order of presentation (eg., a three item set: $(2+5)-2=5, \mathrm{~T} / \mathrm{F}$ ? JOY; $(7-2)+3=6, \mathrm{~T} / \mathrm{F}$ ? LOVE; $(6-4)+7=7, \mathrm{~T} / \mathrm{F}$ ? PEACE; Recall: JOY, LOVE, PEACE). Sets of 2 different lengths (from 3 to 6) were constructed for each affective valence (positive, negative and neutral). Two trials for each set size were presented, with the order of set size varying randomly, so that participants cannot predict the number of items. Additionally, in order to ensure that participants are not trading off between solving the operations and remembering the words, an $85 \%$ accuracy criterion on the math operations is required for all participants. The experimenter transcribes accuracy for math operations on a dedicated protocol. Two training trials precede the task. Stimuli items for the operation span task consisted in 36 arithmetic operations and 36 target words. Words were selected from an affective word database of 200 words developed in our lab and judged by an independent group of 100 students in terms of valence and arousal on a 9-point scale. The 12 positive words had a mean valence of 7.8 (1.5) and a mean arousal level of 5.9 (2.8), the 12 negative words had a mean valence of 2.4 (1.8) and a mean arousal level of 5.9 (2.7). Finally the 12 neutral words had a mean valence of 5.5 (1.9) and a mean arousal level of 2.7 (2.3). All valenced sets were presented to participants, that is, all span levels were administered. The order of the valenced set was counterbalanced across participants. We considered the proportion of the correctly recalled words in the correct serial position by type of stimuli (neutral, positive and negative). For a detailed description and psychometric properties of this task see previous works $[\mathbf{2 , 2 0 ]}$.

\section{Procedure}

Participants were individually tested. After completing a health and demographic questionnaire, they were administered the Ospan test for emotional stimuli.

To limit the influence of sensory variables (sight and hearing) 


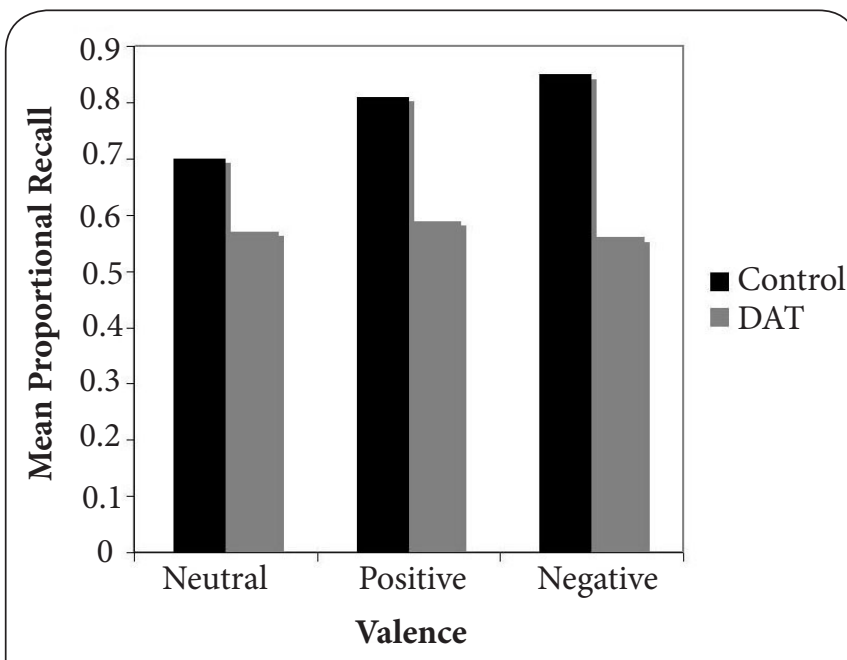

Figure 1. Mean Proportional Recall Scores as a function of Valence and Group.

on test results, the auditory presentation was adjusted to the participants' hearing level. Moreover, for the paper and pencil tasks, all participants were asked whether they found it easy to read the stimuli. All tasks were administrated individually. The order of the tasks within each session was fixed.

\section{Results}

\section{Ospan for emotional words}

Results are presented in Figure 1. A mixed design $2 \times 3$ ANOVA was conducted, with Group (older control and DAT) as between-subject effects, and Valence of stimuli (neutral, positive and negative) as a repeated measure on the mean number of words correctly recalled in serial position calculated as a ratio score. The interaction was decomposed by means of post-hoc pairwise comparisons (Fisher's LSD, all <.05).

With respect to the mean number of words recalled in serial position, results showed main effects of Group, $F(1,38)=22.88$, $p<.001, \eta_{p}{ }^{2}=.06$, and of Valence, $F(2,76)=3.19, p<.05, \eta_{p}{ }^{2}=.02$. The older adults recalled more correct words the DAT adults (.79 vs .59). Moreover, participants recalled fewer correct neutral words (.64) than positive (.70) and negative ones (.71).

The interaction Group X Valence was also significant, $F(2,76)=3.39, p<.05, \eta_{p}{ }^{2}=.02$. Post-hoc comparisons revealed that the older adults recalled more correct positive $(.81)$ and negative words (.85) than neutral ones (.70). Moreover, DAT patients recalled a comparable number of correct positive (.59), neutral (.57) and negative (.56) words.

\section{Discussion}

Much evidence shows how older adults' memory performance benefits when presenting complex tasks with affective stimuli $[2,3]$, but whether DAT patients also show the same benefits is not clear. In fact, declines in general WM performance in DAT patients are well documented [12], while their ability to maintain and manipulate emotional information in WM is less certain [8-11]. The present study examined DAT patients' performance in a WM task, the operation span test, in which affective words were also presented. In line with previous studies $[2,3]$, healthy older adults showed better performance than DAT patients. In addition, with respect to the WM task with emotional stimuli, we replicated the classical emotion enhancement effect in memory [17], as we found a pervasive effect of valence that increased recall in the healthy aging group. In particular, a number of notable findings emerged.

First, we replicated previous findings $[2,3]$ that found a WM emotion enhancement effect in healthy older adults.

Second, most interestingly, our results suggest that emotional stimuli did not influence DAT patients' performance. In fact, valence differences between emotional and neutral words were no longer significant with DAT patients.

With regards to the latter finding, that is, no effect of emotion on WM, our data is in line with numerous findings that reported impaired emotional memory in DAT $[8,9]$. In fact, DAT patients may not benefit from emotional content in their remembering due to amygdala atrophy $[18,19]$. In particular, encoding of emotional material may be disrupted due to a fronto-temporal network dysfunction (amygdalahippocampus-prefrontal cortex circuit) that may, in turn, cause WM deficits for emotional information especially when verbal information is used.

\section{Conclusion}

To summarize, our data point to an emotion modulation of WM performance of healthy older adults during a task that required active maintenance of affective to-be-remembered stimuli in WM. In fact, participants were asked to process a series of arithmetic operations, ignore them and remember only final target words (affective and neutral ones). However, we showed that emotional content does not modulate these active WM functions nor facilitates recall when DAT patients were considered. The novelty of this study lays in the fact that operation span tasks typically better tap our ability to control irrelevant information coming from the affective domain in WM. Future studies should employ this version of the task in order to directly measure WM for emotional material since the affective Ospan can be considered a WM measure that offers specific information regarding individual differences in online emotional information maintenance. This information may also be relevant in clinical settings [20] and can be used to develop adequate protocols $[\mathbf{2 1 , 2 2 ] .}$

\section{Competing interests}

The authors declare that they have no competing interests.

\section{Authors' contributions}

Authors Mammarella and Fairfield contributed to drafting the manuscript. Author Mammarella was both principal investigator and project director of the study. Authors Fairfield, Borella and Caretti designed the experimental protocol. Authors Di 
Mammarella et al. Neuroscience Discovery 2013,

http://www.hoajonline.com/journals/pdf/2052-6946-1-4.pdf

doi: 10.7243/2052-6946-1-4

Domenico was involved in experiment programming and undertook statistical analyses. Authors Di Domenico was involved in data collection and data management. All authors have contributed to and have approved the final manuscript.

\section{Acknowledgement}

No funding was received for this research project. We thank all subjects who participated in our study.

\section{Publication history}

Editor: Armin Zlomuzica, Ruhr-University, Germany.

Received: 21-Mar-2013 Revised: 24-Jun-2013

Re-Revised: 09-Jul-2013 Accepted: 10-Jul-2013

Published: 12-Jul-2013

\section{References}

1. Baddeley AD and Hitch GJ. Working memory. In Bower GH (Ed.), The Psychology of Learning and motivation. 1974; 8:47-89. New York: Academic Press. | Book

2. Mammarella N, Borella E, Carretti B, Leonardi G and Fairfield B. Examining an emotion enhancement effect in working memory: evidence from age-related differences. Neuropsychol Rehabil. 2013; 23:416-28. | Article | PubMed

3. Mikels JA, Larkin GR, Reuter-Lorenz PA and Cartensen LL. Divergent trajectories in the aging mind: changes in working memory for affective versus visual information with age. Psychol Aging. 2005; 20:542-53. Article | PubMed Abstract | PubMed Full Text

4. Borella E, Carretti B and De Beni R. Working memory and inhibition across the adult life-span. Acta Psychol (Amst). 2008; 128:33-44. | Article I PubMed

5. Mammarella N, Fairfield B, De Beni R and Cornoldi C. Aging and intrusion errors in an active visuo-spatial working memory task. Aging Clin Exp Res. 2009; 21:282-91. | Article | PubMed

6. Daneman $M$ and Carpenter PA. Individual differences in working memory and reading. J Verb Lear Verb Beh. 1980; 19:450-466. | Article

7. Engle RW and Kane MJ. Executive attention, working memory capacity, and a two-factor theory of cognitive control. In Ross BH (Ed.), The psychology of learning and motivation. 2004; 44:145-199. New York, NY, US: Elsevier. | Book

8. Kensinger EA, Brierley B, Medford N, Growdon JH and Corkin S. Effects of normal aging and Alzheimer's disease on emotional memory. Emotion. 2002; 2:118-34. | Article | PubMed

9. Kensinger EA, Anderson A, Growdon JH and Corkin S. Effects of Alzheimer disease on memory for verbal emotional information. Neuropsychologia. 2004; 42:791-800. | Article | PubMed

10. Fleming K, Kim SH, Doo M, Maguire G and Potkin SG. Memory for emotional stimuli in patients with Alzheimer's disease. Am J Alzheimers Dis Other Demen. 2003; 18:340-2. | Article | PubMed

11. Satler $\mathrm{C}$ and Tomaz C. Emotional working memory in Alzheimer's disease patients. Dement Geriatr Cogn Dis Extra. 2011; 1:124-38. | Article | PubMed Abstract | PubMed Full Text

12. Mammarella $\mathrm{N}$ and Fairfield $\mathrm{B}$. The role of encoding in reality monitoring: a running memory test with Alzheimer's type dementia. $Q \mathrm{~J}$ Exp Psychol (Hove). 2006; 59:1701-8. | Article | PubMed

13. Crook TH, Bartus RT, Ferris SH, WhitehouseP, Cohen GD, Gershons. Age-associated memory impairment: Pro- posed diagnostic criteria and measures of clinical change-Report of a National Institute of Mental Health workgroup. Developmental Neuropsychology. 1986; 2:261-276. I Article

14. Folstein MF, Folstein SE and McHugh PR. "Mini-mental state". A practical method for grading the cognitive state of patients for the clinician. $J$ Psychiatr Res. 1975; 12:189-98. | Article | PubMed

15. Mondini S, Mapelli D, Vestri A and Bisiacchi PS. Esame Neuropsicologico Breve. 2003; Milano: Raffaello Cortina Editore. | Book
16. Watson D, Clark LA and Tellegen A. Development and validation of brief measures of positive and negative affect: the PANAS scales. J Pers Soc Psychol. 1988; 54:1063-70. | Article | PubMed

17. Mammarella N, Fairfield B and Di Domenico A. Comparing different types of source memory attributes in dementia of Alzheimer's type. Int Psychogeriatr. 2011; 1-8. | Article | PubMed

18. Perrin M, Henaff MA, Padovan C, Faillenot I, Merville A and KrolakSalmon P. Influence of emotional content and context on memory in mild Alzheimer's disease. J Alzheimers Dis. 2012; 29:817-26. | Article | PubMed

19. Klein-Koerkamp Y, Baciu $M$ and Hot P. Preserved and impaired emotional memory in Alzheimer's disease. Front Psychol. 2012; 3:331. | Article | PubMed Abstract | PubMed Full Text

20. Mammarella N, Fairfield B, De Leonardis V, Carretti B, Borella E, Frisullo $E$ and $\mathrm{Di}$ Domenico $A$. Is there an affective working memory deficit in patients with chronic schizophrenia? Schizophr Res. 2012; 138:99-101. | Article I PubMed

21. Mammarella N, Fairfield B and Di Domenico A. When touch matters: an affective tactile intervention for older adults. Geriatr Gerontol Int. 2012; 12:722-4. | Article | PubMed

22. Mammarella $\mathrm{N}$ and Fairfield B. Where did I put my keys? - a 'we' intervention to promote memory in healthy older adults: a controlled pilot study. Gerontology. 2013; 59:349-54. | Article | PubMed

\section{Citation:}

Mammarella N, Fairfield B, Di Domenico A, Borella E and Carretti $\mathrm{B}$. Is working memory affective in dementia of alzheimer's type? Neurosci Discov. 2013; 1:4. http://dx.doi.org/10.7243/2052-6946-1-4 